\title{
Polycythaemia vera: scharfe Behandlung lohnt sich
}

\section{Zur Vermeidung kardiovaskulärer und thrombotischer Ereignisse sollte man bei Patienten mit Polycythaemia vera einen Hämatokrit von unter $45 \%$ anstreben.}

— Die Polycythaemia vera ist eine seltene myeloproliferative Erkrankung, die auf einer Proliferation multipotenter Knochenmarkszellen beruht. Sie führt zu einer abnorm hohen Produktion von erythrozytenähnlichen Zellen und einem erhöhten Hämatokrit. Bei fast allen Patienten findet man erworbene genetische Mutationen. Die wichtigsten Komplikationen und Todesursachen bei der Polycythaemia vera sind Thrombosen, Blutungen und der Übergang in eine Osteomyelofibrose oder akute Leukämie.

Klinische Studien zur Therapie der Polycythaemia vera gibt es nur wenige, und daher steht die wissenschaftliche Evidenz der Leitlinien auf ziemlich tönernen Füßen. Unter anderem lautet eine Empfehlung, der Hämatokrit sollte unter $45 \%$ und die Plättchenzahl unter $400000 / \mu \mathrm{l}$ gehalten werden. In einer randomisierten Studie wurden 365 Erwachsene mit gesicherter Polycythaemia vera mit den Standard-Therapieformen regelmäßige Aderlässe und/oder Hydroxyurea behandelt, wobei in der Studiengruppe die Intensität der Therapie so gewählt wurde, das der Hämatokrit dauerhaft unter $45 \%$ blieb. In der Kontrollgruppe tolerierte man einen Hämatokrit zwischen 45 und $50 \%$.

Nach einem medianen Follow-up von 31 Monaten wurde in der Gruppe mit niedrigem Hämatokrit der primäre Studienendpunkt Tod an kardiovaskulären Ursachen und schwer wiegendes thrombotisches Ereignis bei fünf von 182 Patienten $(2,7 \%)$ erreicht. In der Kontrollgruppe mit höherem Hämatokrit war dies bei 18 von 183 Patienten $(9,8 \%)$ der Fall. Die Progression zur Osteomyelofibrose, Myelodysplasie oder Leukämie erlebten sechs und jeweils zwei Patienten in der Gruppe mit niedrigem Hämatokrit im Vergleich zu 2, 1 und 5 Patienten mit höherem Hämatokrit. Somit betrug die Inzidenz des primären Studienendpunkts in der intensiv behandelten Gruppe 1,1 Ereignisse pro 100 Patientenjahre, im Vergleich zu 4,4 Ereignisse pro 100 Patientenjahre bei den weniger intensiv behandelten Patienten. Dieser Vorteil wurde durch eine erhöhte Rate schwer wiegender hämatologischer Erkrankungen erkauft, die möglicherweise im Zusammenhang mit der höheren Dosierung von Hydroxyurea steht.

\section{- R. Marchioli et al. \\ (Korres.: marchioli@negrisud.it): Cardio- vascular events and intensity of treatment in polycythemia vera. New Engl. J. Med. 2013; 368: $22-33$}

\section{Kommentar}

Die Studie wurde wegen des sich abzeichnenden Erfolgs vorzeitig abgebrochen und war mit 31 Monaten ziemlich kurz. Es könnte sein, dass sich der Trend hin zu malignen hämatologischen Erkrankungen bei längerer Anwendung höherer Dosen von Hydroxyurea noch verstärkt. Für die praktische Durchführung der Therapie sollte man auch nicht vergessen, dass der niedrige Hämatokrit nur mit einer im Durchschnitt um 36\% höheren Zahl von Aderlässen erreicht wurde. Ob Patienten für eine derartige Intensivierung der Therapie, die keine Erleichterung aktueller Beschwerden bringt, sondern auf die Prognose angelegt ist, bei der Stange zu halten sind, erscheint im Einzelfall fraglich. Immerhin darf die Studie für sich in Anspruch nehmen, die Therapie dieser relativ seltenen Erkrankung auf etwas solidere wissenschaftliche Beine gestellt zu haben.

H. S. FÜESSL

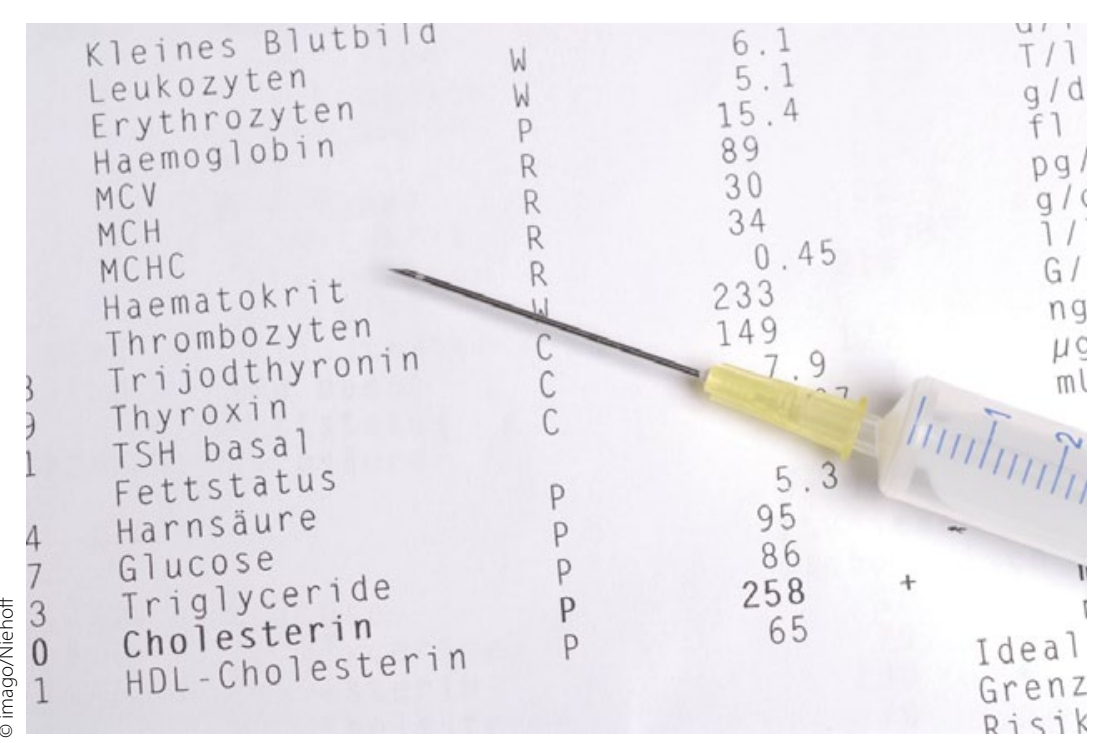

Bei Polycythaemia vera sollte der Hämatokrit 45\% nicht überschreiten. 